\title{
Molecular Tweezer Based on Chenodeoxycholic Acid: Synthesis and Anion Binding Properties
}

\author{
Ki Soo Kim and Iong-Seok Kim \\ Department of Applied Chemistry, Kyngpook National University, Daegu 702-701. Korea \\ Received.Jine 15, 2004
}

Key Words : Molecular tweezer, Chenodeoxycholic acid, l'rea receptor, Anion recognition

The design and synthesis of anion receptors can interact with biologically relevant anions is prominent rescarch field of supramolecular chemistry. "Steroid nucleus is one of the largest rigid and chiral units ubiquitous in nature. Due to the rigidity and preorganized structure of steroid. cholesterol and bile acid derivatives have been studied and shown novel recognition ability for cations. anions. and organic moleeules. ${ }^{2}$ Chenodeoxycholic acid is ideal for lweeser type receptor ${ }^{3}$ design because it contains two hydroxyl groups at $3 \alpha$ and $7 \alpha$ that can be functionalized with a variety of recognition elements thus creating a binding pocket for target guests. Herein we report the first synthesis and anion binding study of molecular tweerer urea receptor based on chenodexoycholic acid.

The synthesis of the receptor 5 is outlined in Scheme 1. The $3 \alpha .7 \alpha$-dihydroxyl groups of chenodeoxycholic acid were transformed to $3 \alpha .7 \alpha$-diamines by three sequential steps. Allylation of 24-tert-butyldimethylsilyloxy-5k-cho- lane-3 $\alpha .7 \alpha$-diol 1 prepared from methyl chenodeoxycholate $^{4}$ in two steps ( $\mathrm{i} / \mathrm{N} \mathrm{Il}_{4}$ reduction, followed by protection with TBSCl) with allyl bromide in the presence of sodium hydride in TIJF resulted in $3 \alpha .7 \alpha$-diallyl compound 2 in $94 \%$ yield. Ilydroboration of the latter with 9-BBN provided the diol 3 in $93 \%$ yield. The $3 \alpha .7 \alpha$-diamino steroid 4 was accessible via the corresponding phthalimide and subsequent hydrazinolysis in $94 \%$ yield. Compound 4 was immediately coupled with phenyl isocyanate in dry ТНГ at room temperalure provided cheno-bis(phenylurea) 5 in $77 \%$ yield.

The anion binding properties of 5 were investigated by ${ }^{1} \mathrm{H}$ NMR titrations in (DCl; solution in the presence of various anions such as $\left.\mathrm{Cl}^{-}, \mathrm{Br}^{-} . \mathrm{I}^{-}, \mathrm{Cl}_{3} \mathrm{CO}_{2}^{-}, \mathrm{H}_{2} \mathrm{PO}_{4}^{-}, \mathrm{NO}_{3}{ }^{-}, \mathrm{IlSO}\right)_{1}^{-}$, and SCN as their tetrabutylammonium (TBA) salt. The addition of equimolar TBACI to a solution of 5 (|TBACI]/5| $-1.0)$ caused significant downfield shifts of both the phenyl and alkyl NH protons by up to $\Delta \delta=1.79$ and $1.08 \mathrm{ppm}$.<smiles>C[C@H](CCCO[Sb])[C@H]1CCC2C3C(CC[C@@]21C)[C@@]1(C)CC[C@@H](O)C[C@]1(C)C[C@H]3O</smiles>

1

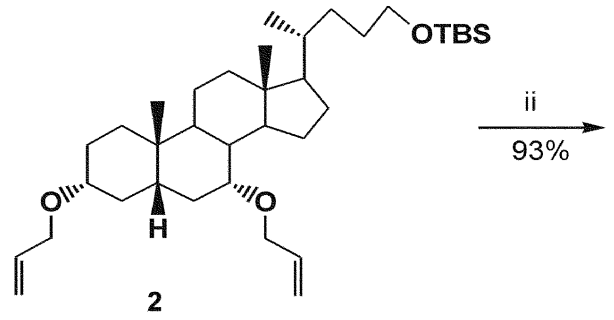

2

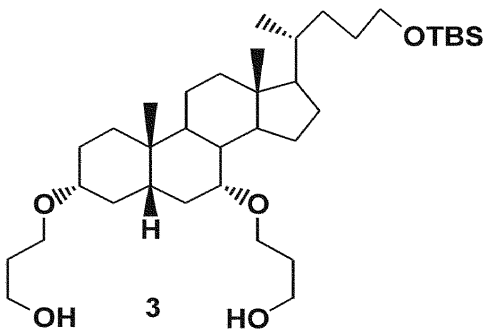<smiles>CC#CCO[Sb](C)(C)C</smiles>

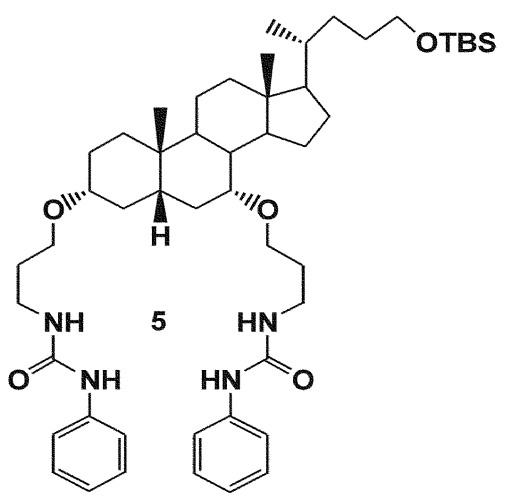

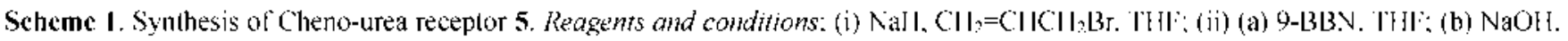
$\mathrm{H}_{2} \mathrm{O}_{2}$; (iii) (a) DE $\mathrm{DD}$. PPh $\mathrm{h}_{3}$. Phthalimide. THF; (b) $\mathrm{H}_{2} \mathrm{NNH}_{2} \cdot \mathrm{H}_{2} \mathrm{O}$. EtOH; (iv) PhNCO, THF.

\footnotetext{
${ }^{7}$ Dedicated to Professor Dong-Han Kim for his $70^{\text {lh }}$ birthday.

“Corresponding Author: Tel: +82-53-950-5588, Fax: $-82-53-950-6594$, e-mail: kimhsok knu.ac.kr
} 


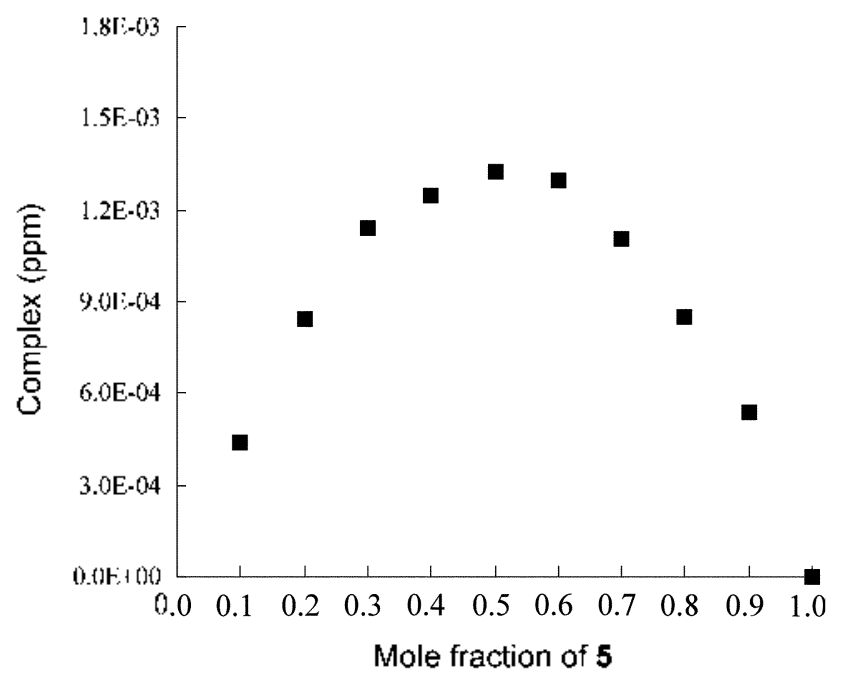

Figure 1. Job plots of cheno-urea receptor 5 with $\mathrm{I}^{1} \mathrm{~A}^{+} \mathrm{II}_{2} \mathrm{PO}_{4}^{-}$in CDCli.

indicating anion binding taking place in the vicinity of urea. As shown in Figure l, Job plot suggests a $1: 1$ stoichiometric complex between 5 and $\mathrm{H}_{2} \mathrm{PO}_{4}{ }^{-}$in $\mathrm{CDCl}_{3}{ }^{5}$

Association constants Ka of $\mathbf{5}$ presented in Table I were calculated by nonlinear curves fitting program EQ-NMR. Cheno-urea 5 binds strongly oxyanions and halides, showing the highest binding affinity $\left(K_{\mathrm{il}}=4,270\right)$ toward dihydrogen phosphate $\left.\mathrm{H}_{2}\right]^{2} \mathrm{O}_{1}^{-}$. In addition, 5 binds $\mathrm{Cl}^{-}$substantially more strongly than $\mathrm{Br}^{-}$. The order of binding affinities in the series of halides $\left(\mathrm{Cl}^{-}>\mathrm{Br}^{-}>I^{-}\right)$is in accordance with that of the basicities.

The signals of the urea protons of 5 in the 'H NMR spectra (on addition of 1 equiv, of salt) appeared at a very low field ( $\delta=9.63$ for $\mathrm{H}_{2} \mathrm{PO}_{1}, \delta=9.04$ for $\mathrm{Cl}^{-}, \delta=8.66$ for $\mathrm{Br}^{-}$, and $\delta$ $=7.39$ for $^{-}{ }^{-}$).

In summary, we have first synthesized and evaluated binding affinities towards anion of molecular tweezer urea receptor based on chenodeoxycholic acid. Further synthesis of other derivatives and binding studies are undergoing in this laboratory.

\section{Experimental Section}

General experimental procedures for melting points. FTIR spectra, mass spectrometry, high resolution MS, elemental analyses, and TLC analysis have been described previously. ${ }^{1} \mathrm{H}$ and ${ }^{1 .} \mathrm{C}$ NMR spectra were recorded on a Varian Unity Spectrometer ('H, $300 \mathrm{MHz}$; ${ }^{13} \mathrm{C}, 75 \mathrm{MHz}$ ) with TMS as an internal standard. ' $\mathrm{H}$ and ${ }^{13} \mathrm{C}$ NMR assignments were made by comparison with spectra of similar sterols. ${ }^{8}$ Flash column chromatography was performed with silica gel Merck silica gel 60 (70-230 mesh). Reactions were carried out under argon atmosphere, and solution was dried over anhydrous sodium sulfate. Chenodeoxycholic acid and other reagents were purchased from either Aldrich or Fluka. Dichloromethane and chloroform were dried over $\mathrm{CaH}_{2}$ and THF was dried over sodium and benzophenone and distilled prior to use. NMR titrations were run at $4.5 \mathrm{mM}$ concentrations, with aliquots of a $0.25 \mathrm{M}(n \mathrm{Bu})_{4} \mathrm{~N}^{+} \mathrm{X}^{-}$salts solution added. The non-linear curve fittings program (EQ-NMR) was used for curve fitting."

Synthesis of 24-tert-Butyldimethylsilyloxy-3 $\alpha, 7 \alpha$-dihydroxy-5 5 -cholane (1). A solution of inethyl chenodeoxycholate $(2.00 \mathrm{~g} .4 .92 \mathrm{mmol})$ in dry THF $(50 \mathrm{~mL})$ was added $\mathrm{LiAlH}_{4}$ (2 eq. $370 \mathrm{mg}$ ) at $00^{\circ} \mathrm{C}$, and stirred for $16 \mathrm{~h}$. The mixture was treated with $10 \% \mathrm{HCl}$ and ethyl acetate. After the precipitated was removed, the filtrate was dried and evaporated to dryness. To a solution of the resulting residue. imidazole (500 $\mathrm{mg}, 7.40 \mathrm{mmol}$ ) and catalytic anount of 4dimethylaminopyridine $(10 \mathrm{mg})$ in dry dichloromethane $(100 \mathrm{~mL})$ and DMF $(10 \mathrm{~mL})$ was added tert-butyldimethylsilyl chloride $(890 \mathrm{mg}, 5.90 \mathrm{mmol})$ in dry dichloromethane $(5 \mathrm{~mL})$ at room temperature. After the reaction was completed, treated with $10 \% \mathrm{HCl}$ and extracted with dichloromethane, dried, and evaporated to dryness. The residue was purified on silica gel chromatography (elution with EtOAc: hexane $1: 3$ ) to give 1: yield 76\%: TLC $R_{f} 0.33$ (EtOAchexane $1: 1)$; mp 92-94 " $\mathrm{C}\left(\mathrm{CH}_{2} \mathrm{Cl}_{2}\right.$-hexane); IR (KBr) $3388,2930,2860,1464,1362,1216,1098,979,836,751$ $\mathrm{cm}^{-1} ;{ }^{1} \mathrm{H}$ NMR $\delta 3.79(\mathrm{~s}, \mathrm{lH}, 3 \beta-\mathrm{H}), 3.52(\mathrm{t}, J=14.7 \mathrm{~Hz}$. $\left.2 \mathrm{H}, 24-\mathrm{CH}_{2}\right), 3.40(\mathrm{~m}, 1 \mathrm{H}, 7 \beta-\mathrm{H}), 0.86(\mathrm{~d}, J=7.2 \mathrm{~Hz}, 3 \mathrm{H}$, $\left.2 \mathrm{~J}-\mathrm{CH}_{3}\right), 0.85$ (s, 9H, $\mathrm{C}\left(\mathrm{CH}_{3}\right)$ ), 0.77 (s, $\left.3 \mathrm{H}, 19-\mathrm{CH}_{3}\right), 0.61$ (s. $\left.3 \mathrm{H}, 18-\mathrm{CH}_{3}\right), 0.03$ (s. $\left.6 \mathrm{H}, \mathrm{Si}\left(\mathrm{CH}_{3}\right)_{2}\right) ;{ }^{12} \mathrm{C}$ NMR $\delta 71.9$, $68.5,63.8,56.0,50.4,42.5,41.4,39.7,39.6 .39 .3 .35 .5,35.3$, $35.0,34.5,32.8,31.9,30.6,29.3,28.2 .25 .9 .23 .6 .22 .8,20.5$, $18.6,18.4,18.3,11.7,-5.3$.

Synthesis of 3,7-diallyl (2): To a solution of $1(2.00 \mathrm{~g}$, $4.06 \mathrm{mmol}$ ) in dry $\mathrm{THF}(100 \mathrm{~mL})$ was added $\mathrm{NaH}(390 \mathrm{mg}$, $16.23 \mathrm{mmol})$, and heated at $60^{\circ} \mathrm{C}$ for $30 \mathrm{~min}$. Allyl bromide ( $1.34 \mathrm{~mL}, 16.23 \mathrm{mmol}$ ) was added to the resulting mixture and heated for $24 \mathrm{~h}$, after that $\mathrm{NaH}(4 \mathrm{eq})$ and allyl bromide (4 eq) was added again and heated for another $24 \mathrm{~h}$. Then the solvent was removed, and the residue was extracted with diethyl ether, washed with brine, dried and evaporated. The residue was purified on column chromatography (elution with $2 \%$ EtOAc-hexane) to give 2: yield 94\%: oil: TLC $R_{f}$ 0.70 (5\% EtOAc-hexane); IR (neat) 2933, 1466, 1379, 1254 , $1096.837 .775 \mathrm{~cm}^{-1} ;{ }^{\prime} H$ NMR $85.86(\mathrm{~m}, 2 \mathrm{H}) .5 .24-5.02(\mathrm{~m}$, $4 \mathrm{H}), 4.08-3.94(\mathrm{~m}, 3 \mathrm{H}), 3.64(\mathrm{dd}, J-12.6,5.7 \mathrm{~Hz}, \mathrm{IH}), 3.52$ (t. $\left.J-6.3 \mathrm{~Hz}, 2 \mathrm{H}, 24-\mathrm{CH}_{2}\right), 3.28(\mathrm{~d}, J-2.1 \mathrm{~Hz}, \mathrm{IH}), 3.1(\mathrm{~m}$, 1H). $0.88\left(\mathrm{~d}, J-7.1 \mathrm{~Hz}, 3 \mathrm{H}, 2 \mathrm{l}-\mathrm{CH}_{3}\right), 0.85\left(\mathrm{~s}, 9 \mathrm{H}, \mathrm{C}\left(\mathrm{CH}_{3}\right)\right)$ ), $0.82\left(\mathrm{~s}, 3 \mathrm{H}, 19-\mathrm{CH}_{3}\right), 0.58\left(\mathrm{~s}, 3 \mathrm{H}, 18-\mathrm{CH}_{3}\right), 0.03(\mathrm{~s}, 6 \mathrm{H}$,

Table I. Association constants of Chen-uread receptor $5^{t}$

\begin{tabular}{ccccccccc}
\hline Anion $\left(\mathrm{X}^{-}\right)$ & $\mathrm{Cl}^{-}$ & $\mathrm{Br}^{-}$ & $\mathrm{I}^{-}$ & $\mathrm{CH}_{3} \mathrm{CO}_{2}^{-}$ & $\mathrm{H}_{2} \mathrm{PO}_{+}^{-}$ & $\mathrm{NO}_{3}^{-}$ & $\mathrm{HSO}_{4}^{-}$ & $\mathrm{SCN}^{-}$ \\
\hline $\mathrm{Ka}\left(\mathrm{M}^{-1}\right)^{h}$ & 2.750 & 1.200 & 260 & 690 & 4.270 & 1.160 & 1.160 & 270 \\
\hline
\end{tabular}

"Isetermined in $\mathrm{Cl})\left(\mathrm{l} \mathrm{l}_{3}\right.$ by ' $\mathrm{H}$ N.MR titration at $298 \mathrm{~K}$. [Host] $=4.5 \times 10^{-3} \mathrm{M}$. "errors estimated to be $\leq 10 \%$ 
$\left.\mathrm{Si}\left(\mathrm{CH}_{3}\right)_{2}\right):{ }^{13} \mathrm{C}$ NMR $\delta 135.8 .116 .1,115.6 .78 .8 .75 .0 .69 .2$. $68.5,63.8,55.9,50.0,42.4,41.8,39.5,35.6,35.3 .35 .0 .33 .5$. $31.9,29.4,28.8,28.2,27.2,26.0,23.7,22.9,20.8$. 18.6. 18.3 . 11.7, -5.3: HRMS (EI) calcd for $\mathrm{C}_{36} \mathrm{H}_{6+} \mathrm{O}_{3} \mathrm{Si}\left(\mathrm{M}^{+}\right)$572.4625. found 572.4639 .

Synthesis of 3,7-diol (3): To a solution of 2 (1.00 g. 1.75 mmol $)$ in dry THF $(100 \mathrm{~mL})$ was added 9-BBN in THF $(0.5$ $\mathrm{M}, 14 \mathrm{~mL}$ ) and stirred at room temperature for $12 \mathrm{~h}$. After the reaction was completed, $20 \% \mathrm{NaOH}(5 \mathrm{~mL})$ and $30 \%$ $\mathrm{H}_{2} \mathrm{O}_{2}(5 \mathrm{~mL})$ was added to the mixture and refluxed for $\mathrm{l} \mathrm{h}$. After the reaction was completed mixture was extracted with ethyl acetate. washed with brine. dried and concentrated to dryness. The residue was purified on column chromatography (elution with EtOAc : hexane $2: 1$ ) to give 3: $93 \%$ yield: oil: TLC $\mathrm{R}_{\mathrm{f}} 0.52$ (EtOAc-hexane $2: 1$ ); IR (neat) 3362, 2934. 1468, 1366, 1253. 1096,837,781 cm ${ }^{-1},{ }^{1} \mathrm{H}$ NMR $\delta 3.7 \mathrm{l}-3.59(\mathrm{~m}, 8 \mathrm{H}) .3 .52\left(\mathrm{t}, J=6.3 \mathrm{~Hz}, 2 \mathrm{H}, 24-\mathrm{CH}_{2}\right)$. $3.23(\mathrm{~s}, \mathrm{lH}) .3 .12(\mathrm{~m}, \mathrm{lH}) .0 .87\left(\mathrm{~m} .6 \mathrm{H}, 19-\right.$ and $\left.21-\mathrm{CH}_{3}\right)$. 0.84 (s, $\left.9 \mathrm{H}, \mathrm{C}\left(\mathrm{CH}_{3}\right)_{3}\right) .0 .58$ (s. $\left.3 \mathrm{H} .18-\mathrm{CH}_{3}\right) .0 .03$ (s. $6 \mathrm{H}$. $\left.\mathrm{Si}\left(\mathrm{CH}_{3}\right)_{2}\right):{ }^{13} \mathrm{C}$ NMR $\delta 79.5,76.3 .67 .0,66.3 .63 .7,62.1$. $61.1,56.0,50.3,42.4,41.7,39.5,39.3,35.5,35.2 .35 .0 .33 .6$. $32.6,32.1,31.8,29.5,28.5,28.1,27.2,25.9,23.7 .22 .8 .20 .7$. 18.6, 18.3. 11.6. -5.3. FAB-MS calcd for $\mathrm{C}_{36} \mathrm{H}_{68} \mathrm{O} \mathrm{O}_{5} \mathrm{Si}$ : 608.48 , found: $\mathrm{m} / \mathrm{z} 609(\mathrm{M}+\mathrm{H})^{+}$.

Synthesis of 3,7-diamine (4): After a mixture of 3 (500 mg. $0.82 \mathrm{~mm}$ ol). phthalimide $(710 \mathrm{mg}, 4.1 \mathrm{mmol}$ ) and triphenyl phosphine $(1.00 \mathrm{~g} .4 .1 \mathrm{mmol})$ was stirred in dry THF $(50 \mathrm{~mL})$ at room temiperature, diethyl azodicarboxylate $(0.63 \mathrm{~mL} .4 .1 \mathrm{mmol})$ was added and continued to stir. After the solvent was removed. and the residue was extracted with ethyl acetate. washed with brine, dried. and concentrated. Without further purification, the residue and hydrazine monohydrate $(410 \mathrm{mg} .8 .2 \mathrm{mmol})$ was refluxed in ethanol $(200 \mathrm{~mL}$ ) for $24 \mathrm{~h}$. Then the solvent was removed. extracted with diethyl ether. washed, dried. and concentrated. The residue was purified on colunu cluromatography (elution with $\left.\mathrm{CH}_{2} \mathrm{Cl}_{2}: \mathrm{MeOH}: \mathrm{NH}_{4} \mathrm{OH} 16: 3: 0.5\right)$ to give $4: 94 \%$ yield: oil; $\mathrm{TLC} \mathrm{R}_{\mathrm{f}} 0.39\left(\mathrm{CH}_{2} \mathrm{Cl} \mathrm{Cl}_{2}-\mathrm{MeOH}-\mathrm{NH}_{4} \mathrm{OH} 16: 3: 1\right)$ ) IR (neat) 2934, 2861, 1466, 1364, 1254, 1099, 837. 775. 737 $\mathrm{cm}^{-1}$. ${ }^{1} \mathrm{H}$ NMR (400 MHz) $\delta 3.63-3.46(\mathrm{~m} .6 \mathrm{H}), 3.19$ (s. lH). $3.05(\mathrm{~m} .1 \mathrm{H}) .2 .75(\mathrm{~m} .4 \mathrm{H}) .0 .87(\mathrm{~d} . J=7.1 \mathrm{~Hz}, 3 \mathrm{H} .2 \mathrm{l}-$ $\mathrm{CH}_{3}$ ). 0.87 (s. $\left.3 \mathrm{H} .19-\mathrm{CH}_{3}\right) .0 .85$ (s. $\left.9 \mathrm{H} . \mathrm{C}\left(\mathrm{CH}_{3}\right)_{3}\right) .0 .58$ (s. $\left.3 \mathrm{H} .18-\mathrm{CH}_{3}\right) .0 .03$ (s. $\left.6 \mathrm{H} . \mathrm{Si}\left(\mathrm{CH}_{3}\right)_{2}\right):{ }^{13} \mathrm{C} \mathrm{NMR}(125 \mathrm{MHz}) \delta$ 79.3. 75.9.66.2.65.8.63.7.56.0.50.2. 42.3.41.8. 39.7.39.5. $39.4,35.5,35.3,35.2,350,33.8,33.6,33.5,31.8 .29 .5 .28 .6$. $28.1,27.3,25.9,23.7 .22 .8,20.7,18.6 .18 .3,11.6,-4.9$. MS (relative intensity. \%) $\mathrm{m} / \mathrm{z} 607\left(\mathrm{M}^{+}, 3\right)$.

Synthesis of cheno-urea (5): A solution of $4(100 \mathrm{mg}$. $0.165 \mathrm{mmol})$ in dry THF $(10 \mathrm{~mL})$ was reacted with phenyl isocyanate $(0.09 \mathrm{~mL} .0 .825 \mathrm{mmol})$ at room temperature for 2 h. Then the solvent was removed. extracted with ethyl acetate. washed. dried. and concentrated. The residue was purified on column chromatography (elution with EtOAchexane $1: 3$ ) to give 5 yield $92 \%$; TLC $R_{i} 0.50$ (EtOAChexane $1: 1$ ); $\operatorname{mp} 95.96{ }^{\circ} \mathrm{C}$ : IR ( $\left.\mathrm{KBr}\right) 3335.2934,2863$. 1649. 1559. 1501, 1443. 1312, 1244, 1098. $756.694 \mathrm{~cm}^{-1}$; ${ }^{1} \mathrm{H}$ NMR $\delta 7.85(\mathrm{~s} . \mathrm{lH}) .7 .66(\mathrm{~s}, \mathrm{lH}) .7 .37 .7 .3 \mathrm{l}(\mathrm{dd}, J=15.3$, $7.8 \mathrm{~Hz}, 4 \mathrm{H}) .7 .26-7.15(\mathrm{~m}, 6 \mathrm{H}) .6 .7(\mathrm{~s}, \mathrm{lH}) .6 .43(\mathrm{~s} . \mathrm{H})$, $3.72 .3 .39(\mathrm{~m} .10 \mathrm{H}) .3 .16(\mathrm{~s} . \mathrm{lH}) .3 .04(\mathrm{~m} . \mathrm{lH}) .0 .86(\mathrm{~d} . J=$ $\left.7.2 \mathrm{~Hz} .3 \mathrm{H} .21-\mathrm{CH}_{3}\right), 0.85$ (s. $\left.9 \mathrm{H} . \mathrm{C}\left(\mathrm{CH}_{3}\right)_{3}\right) .0 .78$ (s. $3 \mathrm{H}, 19-$ $\mathrm{CH}_{3}$ ). 0.58 (s. $3 \mathrm{H}, 18-\mathrm{CH}_{3}$ ). 0.03 (s. $6 \mathrm{H}, \mathrm{Si}\left(\mathrm{CH}_{3}\right)$ ) $) .{ }^{13} \mathrm{C}$ NMR $\delta 156.6,156.4,139.3,129.0,128.9,123.0,122.6$. 120.1, 119.6. 79.3. 76.2. 66.6. 65.8. 63.8. 56.2. 50.6, 42.5 . $41.6 .39 .7 .39 .4 .38 .5 .35 .6 .35 .2 .33 .9 .31 .9,30.5,30.0,29.5$, 28.8. 28.2, 27.5, 26.0. 23.8, 22.9, 20.8, 18.7, 18.4. 11.7. -5.3; FAB-MS calcd for $\mathrm{C}_{5 i \mathrm{i}} \mathrm{H}_{8 i \mathrm{~N}} \mathrm{~N}_{4} \mathrm{O}_{5} \mathrm{Si}: 844.59$, found: $\mathrm{m} / \mathrm{z} 845$ $(\mathrm{M}+\mathrm{H})^{-}$: Anal. Calcd for $\mathrm{C}_{3 i} \mathrm{H}_{8 i j} \mathrm{~N}_{4} \mathrm{O}_{5} \mathrm{Si}: \mathrm{C}, 71.05 ; \mathrm{H}, 9.54$ $\mathrm{N}, 6.63$. Found $\mathrm{C}, 70.87$; H. 9.57 : N, 6.50

Acknowledgements. This work was supported by grant No. R05-2001-000-00227-0 from Korea Science \& Engineering Foundation and Brain Korea 2l Project.

\section{References}

1. (a) Supromolecular Chemisny of Anions: Bianch. A.: BowmanJames. K:: García-España. E.. Eds: Wiley-VCH: New York: 1997. (b) Gale. P. A. Coord Chem. Rer: 2003. 240. 191. (c) Beer. P. D.: Gale, P. A. Angew: Chent. Int Ed. 2001, t0. 486. (d) Antonisse, M. M. G.: Reinhoudt, D. N. Electroandlysis 1999, 11. 1035. (e) Bühlmann, P.; Pretsch. E.: Bakker. E. Chem. Ren 1998. 98. 1593. (f) Schmidtchen. F. P.: Berger. M. Chom. Rk? 1997. 97. 1609. (g) Kang. Y. S.: Ko. S. W.: Song. I. H.: Ryu. B. J.: Nam. K. C. Bull Korean Chem Soc. 2003. 24.681.

2. For reviews, see: (a) Davis, A. P. Joos. J.-B. Coord Chem. Rer: 2003. 240. 143. (b) Wallimann, P.: Marti. T.: Fürer, A.: Diederich, F. Chem. Rev: 1997, 97. 1567. (c) Davis. A. P. Gilmer, J. F. Perry: J. T. Angew: Chem. Int Ed. Engl. 1996. 35. 1312. (d) Daqis. A. P. Chent. Soc. Rev: 1993. 22. 243.

3. For selected examples of steroid based tweezers. see: (a) Shimn. I. H.; Jeong. I. S.: Lee, M. H.; Hong, H. P.: On. J. H.: Kim, K. S. Kim. H.-S: Kim. B. H.: Cha, G. S.: Nam, H. Talanta 2004, 63.61. (b) Kim, B. H.: Lee, C. S.: Shim, J. H.: Hong, H. P. Cha. G. S. Jun1. Y. M.: Nam. H. Talanta 2003. 61. 393. (c) Ayling. A. T.: Pérez-Payán. M. N.: Davis. A. P. J. Am. Chem. Soc 2001. 123. 12716. (d) Potluri. V. K.: Maitra. U. J. Org. Chent. 2000. 65. 7764 (e) Pyun. H. J.; Chu. J.: Jun. Y. M.: Kim. D. J. Bull. Koream Chent. Soc, 1999, 20, 112 .

4. Savage. P. B: Allman, G. W. Willardson, B. M: Driscoll. C. D.: Budge. L. P: Li. C. J. Am. Chem. Soc. 1999. 121.931.

5. (a) Job. P. Compt. Rend 1925. 180. 928. (b) Blanda. M. T: Horner. J. H.: Newcomb. M. J. Org. Chem. 1989. 5t. 4626.

6. Hynes. M. J. J. Chem. Soc. Daton Trams. 1993, 311.

7. Kim. H.-S: Choi. B.-S: Kwon. K.-C: Lee. S.-O.: Kwak, H. J.; Lee. C. H. Biong 1 led. Chem. 2000.8. 2059

8. Blunt. T. W.: Stothers. T. B. Org Mfag Reson. 1977. 9. 439. 